\title{
El adobe: factores históricos gatillantes en la pérdida de la cultura constructiva en Chile
}

\section{The adobe: historical factors triggering the loss of construction culture in Chile}

DOI: $10.46932 / \mathrm{sfjdv2n2-017}$

Received in: january 1st, 2020

Accepted in: March 30th, 2020

\author{
Rodrigo Pérez \\ Facultad de Arquitectura, Urbanismo y Geografía, Universidad de Concepción - Red ARCOT Cátedra \\ UNESCO Chile, Chile \\ E-mail: rodrigofperez@udec.cl
}

\section{RESUMEN}

Chile, presenta una larga geografía y una diversidad climática que ha condicionado históricamente la respuesta local arquitectónica y constructiva, adaptándo las soluciones a los recursos naturales disponibles. Entre los sistemas constructivos con tierra, el Adobe ha sido el más utilizado pero también el más cuestionado por su fragilidad a los movimientos telúricos y por tanto a la seguridad que ofrece. Frases como "el adobe mata" o "el adobe es peligroso", reflejan algunos de los prejuicios que existen contra este material y que desgraciadamente no sólo están en la mente del ciudadano común sino también en la opinión (desinformada) de profesionales, autoridades políticas, etc. Esto da pié a esta investigación, para encontrar respuesta a ¿Qué ha sucedido a lo largo de la historia chilena que ha llevado a este noble material de ser el más utilizado desde épocas pre-hispánicas al más despreciado en la actualidad?. El objetivo principal es buscar y dar a conocer las razones y acontecimientos para rescatar y revalorar el Adobe, rompiendo con algunos mitos y paradigmas muy arraigados en nuestra sociedad actual que ponen freno al uso del material tierra y al desarrollo de técnicas innovadoras con este material tan noble, ecológico y sustentable. En una primera etapa, se realiza un análisis histórico para descubrir la base de estos saberes y técnicas locales en torno al Adobe, estableciendo una cronología de los hechos importantes que fueron gatillando los cambios para finalizar en una segunda etapa con un análisis comparativo del estado del arte de las construcciones que a la fecha están en pié y en buen estado de conservación. La investigación esta en etapa inicial pero ya se vislumbran algunos resultados que dan luces del origen del problema, no sólo socio-culturales y comunicacionales, sino también técnicos, condicionados por políticas

Palabras clave: cultura constructiva, historia, construcción con tierra, normativa, adobe.

\begin{abstract}
Chile has a long geography and a climatic diversity that has historically conditioned the local architectural and constructive response, adapting the solutions to the available natural resources. Among the earth construction systems, Adobe has been the most used but also the most questioned due to its fragility to telluric movements and therefore to the safety it offers. Phrases such as "adobe kills" or "adobe is dangerous" reflect some of the prejudices that exist against this material and that unfortunately are not only in the minds of the common citizen but also in the (uninformed) opinion of professionals, political authorities, etc. This gives rise to this research, to find an answer to the question: What has happened throughout Chilean history that has taken this noble material from being the most used since pre-Hispanic times to the most despised at present? The main objective is to seek and make known the reasons and events to rescue and revalue Adobe, breaking with some myths and paradigms deeply rooted in our current society that put a brake on the use of earthen material and the development of innovative techniques with this noble, ecological and sustainable material. In a first stage, a historical analysis is made to discover
\end{abstract}


the basis of these local knowledge and techniques around Adobe, establishing a chronology of the important events that triggered the changes to end in a second stage with a comparative analysis of the state of the art of the constructions that are still standing and in a good state of preservation. The research is in its initial stage, but some results are already emerging that shed light on the origin of the problem, not only socio-cultural and communicational, but also technical, conditioned by policies.

Key words: building culture, history, earth construction, regulations, adobe.

\section{INTRODUCCIÓN}

A lo largo de la historia de Chile, desde épocas pre hispánicas, se han sucedido una serie de hechos o circunstancias de distinta índole (históricos, naturales, sociales, políticos, económicos, culturales, etc.) que han sido factores gatillantes en la transformación de la técnica constructiva del Adobe llegando en los tiempos actuales a una pérdida del saber hacer ancestral. Por una parte está el legado ancestral de los pueblos originarios y luego, con el descubrimiento de américa, se produce un hito importante que determina un antes y un despues en nuestra historia constructiva, comienza el período de la conquista española y surgen las primeras ciudades como las conocemos hoy en día. La arquitectura urbana sufre transformaciones en la medida en que se van desarrollando las ciudades en sus distintos ámbitos; económico, demográfico, político, etc. y en una constante busqueda identitaria con el momento que se vive en cada época. Sumado a lo anterior, la llegada de nuevos materiales y sistemas constructivos productos del desarrollo industrial, tecnológico, modas, tendencias y acompañado permanentemente por grandes sismos y sus consecuentes efectos de destrucción, que siempre han abierto la oportunidad a la renovación. Es aquí donde se centra la primera mirada en esta busqueda.

\section{OBJETIVOS}

El objetivo principal de esta investigación es dar respuesta a una interrogante que de alguna manera encierra una preocupación actual sobre las razones que han llevado por una parte a prohibir por ley el uso del adobe como sistema constructivo y por otra a rechazarlo como alternativa viable.

¿Qué ha sucedido a lo largo de la historia chilena que ha llevado a este noble material de ser uno de los más utilizado desde épocas pre-hispánicas al más despreciado en la actualidad?.

Investigar y recopilar acontecimientos y experiencias que se han sucedido históricamente resultan necesarias para entender y no repetir errores en las toma de decisiones referidas al adobe y así poder reeducar a las nuevas generaciones y contribuir a poner nuevamente en valor sus extraordinarias propiedades para quitar el freno al uso del material tierra y al desarrollo de técnicas innovadoras con este material tan noble, ecológico y sustentable. 


\section{ESTRATEGIA METODOLÓGICA}

El trabajo de investigación se propone en dos etapas; la primera, que se expone en este artículo y que a la fecha está en pleno inicio y en donde se está realizando un análisis bibliográfico y documental histórico para descubrir y rescatar la base de estos saberes y técnicas locales en torno a la arquitectura en tierra, especificamente con respecto al Adobe en Chile y establecer una seguimiento cronológico de los hechos importantes que fueron gatillando los cambios en las técnicas constructivas sobre la base de los permanentes eventos telúricos a lo largo del territorio, sumandole a estos en el proceso aquellos otros hechos de relevancia que, directa o indirectamente, también influyeron en estos cambios.

Debido a la gran cantidad de bibliografía revisada y sobre todo por revisar y el tiempo que esto demanda, los resultados de los primeros análisis muestran que es particularmente relevante el punto de vista de la cronología de los movimientos telúricos, los que al fin y al cabo son los grandes modeladores y sobre todo promotores de los cambios en las ciudades y su arquitectura.

Finalmente, en una segunda etapa, se realizará una caracterización evolutiva de la técnica constructiva del adobe con toda su metamorfósis sufrida desde sus origenes para terminar con un levantamiento y análisis arquitectónico - constructivo en edificaciones de adobe de al menos tres localidades de la región del Biobío, que por su data fundacional puedan mostrarnos a través de sus testimonios arquitectónicos aún en pié, el estado del arte de las construcciones locales en adobe en la actualidad. La decisión de elegir localidades semi rurales, de pequeños pueblos, es porque en ellos no se habrían producido los cambios que si se evidencian en las grandes ciudades asi que es más probable encontrar en estos poblados edificaciones que se hayan mantenido más o menos intactas desde sus fechas de construcción. Esto permitirá detectar y evaluar si sufrieron cambios o no y analizar el por qué aún se mantienen en pié y así intentar dar respuestas más concluyente a esta investigación.

\section{RESULTADOS}

A pesar de encontrarse en una etapa inicial la investigación, presenta algunos resultados que dan luces de algunos hechos o situaciones no sólo socio-culturales y comunicacionales, sino también técnicos, condicionados por políticas económicas que sistemáticamente fueron restringiendo el uso del adobe hasta llegar casi a eliminarlo.

\subsection{CHILE PAÍS SÍSMICO Y LOS TERREMOTOS COMO MODELADORES CONSTRUCTIVOS}

Sin duda al referirnos a Chile, no podemos dejar de lado uno de los factores más importantes en la transformación e innovación de las ciudades y por lo tanto de la arquitectura y sus soluciones constructivas locales desde sus inicios, se trata de los terremotos. Chile es a nivel mundial uno de los paises con los 
terremotos más fuertes y devastadores que se tenga registros (Figuras 1 y 2). Los primeros registros chilenos datan de 1570 plasmados en algunas crónicas de la época o relatos transmitidos de una generación a otra en la intimidad del núcleo familiar.

Figura 1. Últimos terremotos históricos según liberación de energía
Figura 2. Los peores terremotos en la historia de Chile desde 1900
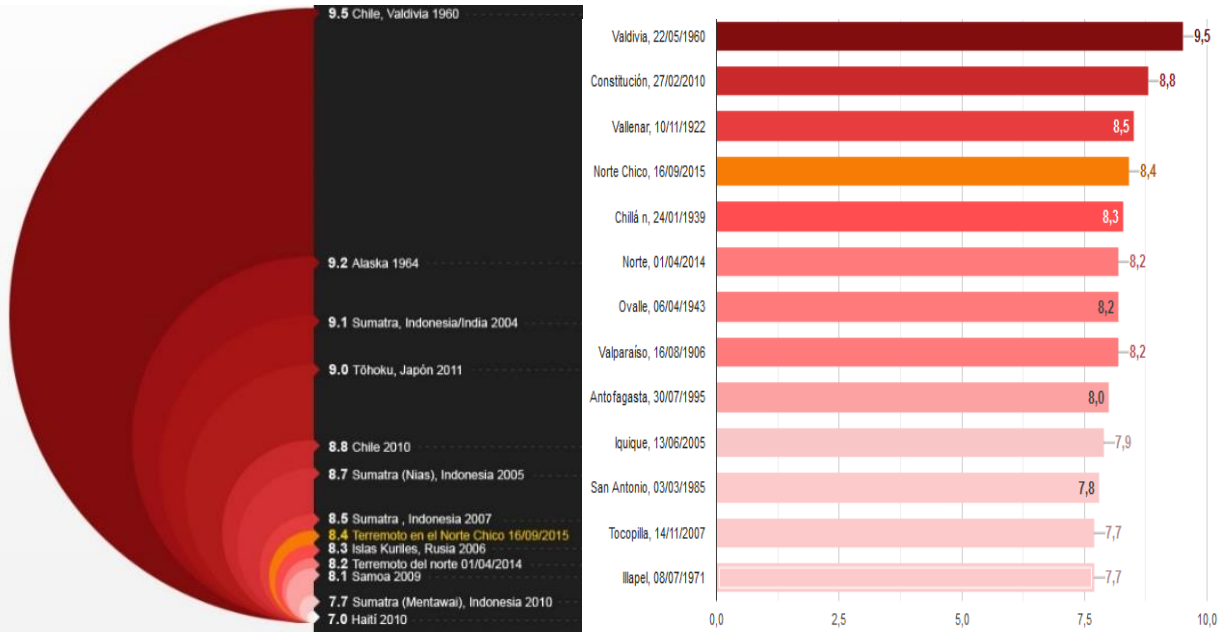

(Fuente: Centro Sismológico de la Universidad de Chile)

Parece lógico a primera vista responsabilizar a los terremotos por el daño y colapso de las edificaciones, más aún si se trata de adobe, pero si los sismos siempre han estado aquí habría que preguntarse entonces ¿Qué ha pasado en todos estos años?. Será que somos nosotros los que no hemos sabido dar en el tiempo las respuestas adecuadas. También sería importante preguntarse ¿Qúe respuesta ante el sismo deberíamos esperar de una edificación?. De acuerdo a la experiencia personal en asesorías post $27 \mathrm{~F}$ en la ciudad de Concepción, se visitaron a más de un centenar de familias afectadas de diferentes estratos socio - económico - cultural. Al ser entrevistados, una gran mayoría coincidió en que la espectativa de respuesta de sus construcciones ante el terremoto ( 8,8 Rischter) fueron que sus viviendas resistieran y no se agrietaran y menos colapsaran. Esto deja entre ver un cierto desconocimiento de cual es el espíritu o filosofía detrás de las Normas actuales. Según Benjamín Navarrete, Constructor Civil y académico en la Escuela de Construcción Civil de la Pontificia Universidad Católica de Chile, afirma que en la actualidad se sigue la siguiente filosofía: "Para sismos de baja intensidad no se producirán daños; sismos de mediana intensidad se aceptarán daños en elementos y componentes no estructurales; sismos de gran intensidad, se pueden producir daños en elementos estructurales, pero en ningún caso se producirá el colapso de la estructura", en otras palabras podríamos decir que la finalidad de la Norma sísmica es, más que salvar la edificación, resguardar la vida de sus moradores ante un evento sísmico, por lo tanto es esperable que dependiendo de la magnitud del evento, si se puedan producir grietas con menor o mayor compromiso estructural. Si llevamos esta inquietud atrás en el tiempo, las espectativas en las personas, 
según se deduce de algunos relatos, habrían sido sino iguales, muy similares, atribuyéndole a la aparición de grietas un daño irreparable, como cuando se quiebra una copa de cristal, pero desconociendo que en la arquitectura en general y particularmente el adobe existe un alto porcentaje de daños reparables. Es así como hasta en la actualidad para una persona común, por su normal desconocimiento técnico del coportamiento sísmico de una edificación, la presencia de una grieta luego de un movimiento telúrico, la asocia inmediatamente con una falla estructural sin detenerse a observar que talvez la grieta es solo de elementos no estructurales como por ejemplo el revoque o estuco.

Los constantes movimientos telúricos característicos de nuestro país, a lo largo de nuestra historia y extensa geografía con su diversidad de climas, relieves, etc. han obligado a sus habitantes en las diferentes latitudes, "a desarrollar una serie de estrategias de sismoresistencia que, a pesar de no poseer un registro escrito, pueden leerse claramente en la forma urbana, en el diseño arquitectónico y en las características constructivas de los edificios” (como se cita en Jorquera, 2014. p.2)

Solo revisando cronologicamente los innumerables sismos que han sacudido al país desde que se tienen registro, podemos observar circunstancias de distinta índole que aportan a la comprensión y dan en parte algunas respuestas al planteamiento del problema. No pasa lo mismo si siempre vemos los eventos en forma aislada, recordemos que la naturaleza es cíclica por ende los eventos telúricos también lo son, por lo tanto, si no aprendemos de los errores del pasado, los seguiremos repitiendo en el futuro. De alguna manera eso es lo que ha venido sucediendo en Chile, luego de cada evento sísmico y con el consiguiente daño y detrucción que este produce, las autoridades edilicias, ministeriales, cuerpo de bomberos, profesionales, etc. salen a las calles a evaluar los daños y determinar rápidamente que edificaciones están en condiciones de habitabilidad y cuales no, muchas veces por no decir la mayoría, las inspecciones han sido meramente visual y en el caso de las construcciones de adobe, sin el conocimiento técnico adecuado, no se han tomado el tiempo suficiente para evaluar realmente su estado y guiados por intereses de desarrollo local, reactivación económica, etc., se han toman decisiones de demolición sin argumentos suficientes y sin saber el origen del problema que muchas veces es producto de malas prácticas constructivas y no del sistema constructivo en si. Si a lo anterior, sumamos hoy en día la rapidez mediática de la información, entonces el mensaje que llega a la persona común acrecienta el prejuicio sobre la construcción con adobe cuyas imágenes son las primeras y casi únicas en mostrar como testimonio de la destrucción. 
Figura 3. Album Gráfico del Terremoto del Norte, M.J. Ortíz, 1923.
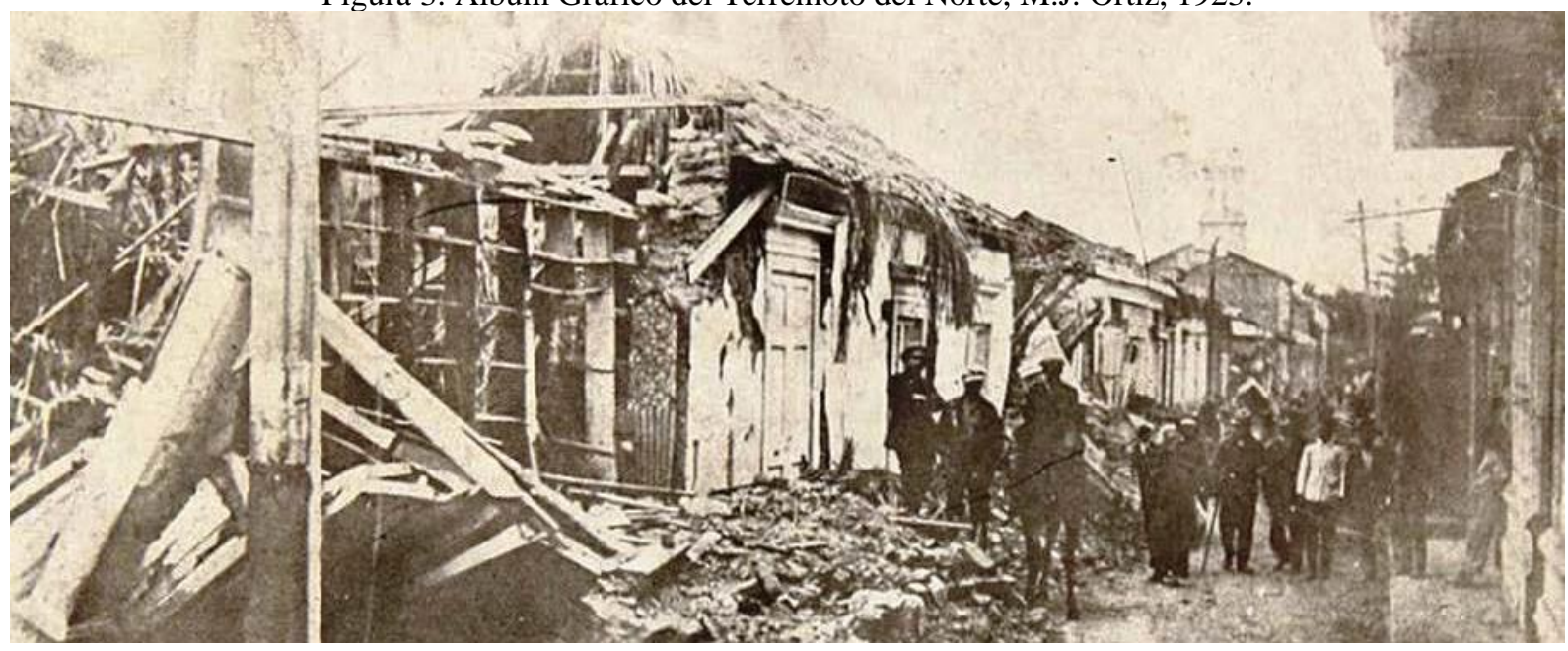

\subsection{INTERCAMBIOS E INFLUENCIAS CONSTRUCTIVAS EN EL PERÍODO PRE-HISPÁNICO}

Ya se mencionó que la llegada de los españoles marco un antes y un despues en general en la historia americana. Entonces, para poder abordar el tema, se hace necesario dar una mirada a la situación de intercambios o influencias culturales - constructivas que se produjeron antes de la llegada de los españoles y que pudieron modelar y talvez transformar la manera de construir de los autóctonos chilenos (materialidad, sistema constructivo, criterios sísmicos).

Sin duda, en sudamérica la cultura que llegó a construir un gran imperio, vigente a la llegada de los españoles, fueron los Incas. Como producto de su proceso de expansión y conquista, una buena parte del territorio chileno formaba parte del "Collasuyo", que correspondía al distrito sur, uno de los cuatro en que se dividía el Imperio tomando como centro la ciudad de Cuzco y llegando hasta el río Maule en la actual séptima región de Chile. "Entre las clases sociales estaban los Mitmaqkuna, pobladores que eran trasladados para colonizar otras regiones junto con sus familias bajo el mando de su jefe étnico" (Vergara, 2000). Aquí encontramos algunos indicios de influencia cultural debido a que una de las funciones de los Mitmaqkuna por mandato supremo, era la de transmitir a los pueblos conquistado y dominados los aspectos propios de la Cultura Inca.

La arquitectura de las casas incas varió mucho en el Imperio dependiendo entre otras cosas del clima donde estaban ubicadas. Las construcciones habitacionales se podrían dividir en dos grandes grupos según su ubicación; las cordilleranas (de la sierra) y las de la costa. "Las casas cordilleranas fueron siempre de piedra, con sus techos de paja a dos aguas, acaso una sola puerta pequeña y a veces ninguna ventana." Por otra parte estaban las casas de la costa algo diferentes a las anteriores sus "paredes eran muy gruesas, de barro o adobe, tenían varias puertas y ventanas; los techos eran planos por ser vigas de huarango. Estos travesaños sostenían tupidos entrelazados de caña perceptibles en el interior de la habitación, no así desde 


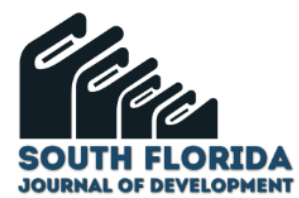

el exterior por estar cubiertos por una capa de barro seco mezclado con paja.” (del Busto, 1981, p. 274 y 276)

Si damos una mirada a la arquitectura de los pueblos altiplánicos (Aymaras y Quechuas) en el norte de Chile (Figuras 4 y 5), reconocemos rapidamente las características descritas en el párrafo anterior y que han perdurado en el tiempo hasta la actualidad con un mínimo de alteraciones, principalmente en su materialidad debido a que los poblados estan dispersos en un gran territorio y lejos de las grandes ciudades. Las transformaciones propias de la modernidad no han llegado con tanta fuerza, manteniendo vigente y evidente la influencia Inca.
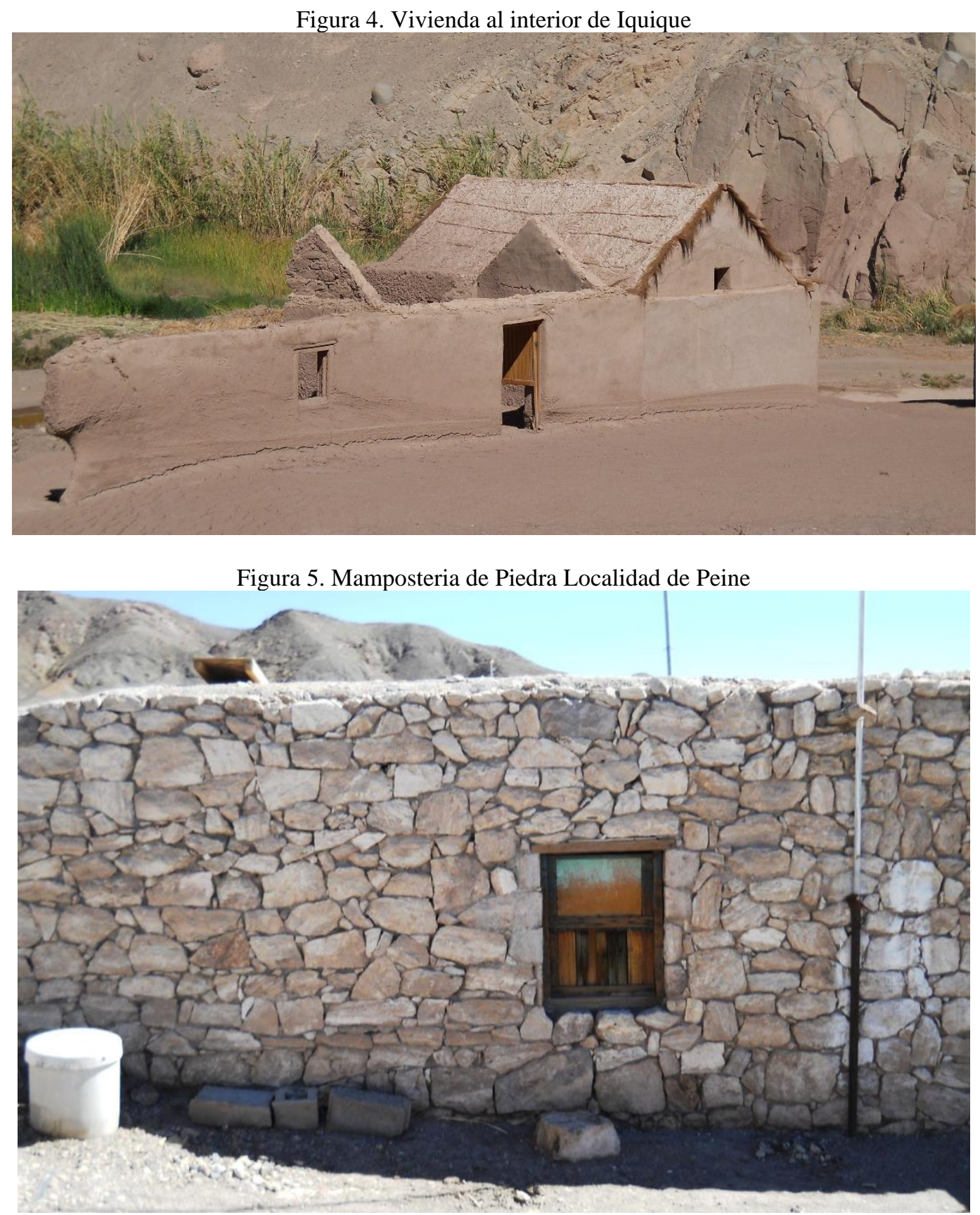

Fuente propia 
Entre los pueblos originarios pre hispánicos de la zona centro-sur, se encontraban los Picunches, Mapuches, Huilliches y Pehuenches. Habitaron entre los ríos Aconcagua y el Itata. Los Picunches (del mapudungun: pikun che, "gente del norte"), era el pueblo perteneciente a la zona central de Chile llamada Pikun Mapu. Producto de su contacto con los Diaguitas, y posteriormente con los Incas, este pueblo alcanzó un mayor desarrollo que los Mapuches que se emplazaban mas al sur.

El pueblo Picunche actualmente está extinto, producto de muertes por nuevas enfermedades, guerras y genocidio en el período de la conquista española, pero tambien existió un abandono de su cultura al integrarse a la cultura española produciendose el mestizaje.

\subsection{TERREMOTOS Y PRECARIEDAD CONSTRUCTIVA DURANTE EL SIGLO XVI}

Luego del descubrimiento de Chile con la expedición de Hernando de Magallanes en 1520 y con la de Diego de Almagro en 1536, concluye con la llegada de Pedro de Valdivia en 1541, quien inicia el período de la Conquista. Ya en los inicios de la Conquista, Barros Arana (2000, p.282) refieriendose a la ciudad de Santiago señala que [...]“el aspecto de aquella ciudad de adobones y de paja, que sin embargo, se llama la capital del reino de la Nueva Extremadura, debió ser el de las más miserables aldeas." dejando en claro la precaria materialidad de sus construcciones pero también nos señalas el uso de la tierra y fibras en ellas (paja).

Por otra parte, al parecer, no existen registros de eventos telúricos sino hasta en 1562 y 1570 donde se producen sendos terremotos en la ciudad de Concepción y 1575 en Valdivia. Sin embargo, Amunátegui (1882, p. 526 y 527) citando a el maestre de campo Alonso González de Nájera, quien señala que "Todo el reino de Chile es sujeto a terremotos," por lo cual para los nativos son comunes los temblores y refiriendose a los edificios, acota que [...] "por lo que los fabrican jeneralmente bajos", es decir en un piso, aludiendo a un gran terremoto que asoló la ciudad de Concepción en el año 1562 en su antiguo emplazamiento, donde actualmente se ubica la ciudad de Penco. Vale la pena destacar que en este período las construcciones son más efímeras, expuestas a los permanentes ataques y destrucción por parte de los indigenas solo se construye con madera (troncos) y fibras (paja). 


\title{
4.4 HITOS Y NECESIDAD DE TOMA DE CONCIENCIA DE SEGURIDAD
}

\section{CONSTRUCTIVA A PARTIR DEL SIGLO XVII}

Dada la larga lista de terremotos, se presentan acá solo aquellos en donde la revisión bibliográfica y documental, hasta el momento, a permitido encontrar antecedentes que aporten al análisis.

- 13 de mayo de 1647. Tiene lugar el mayor terremoto registrado en las crónicas Coloniales y que redujo a escombros la ciudad de Santiago. Este hecho catastrófico deja a la luz uno de los primeros hechos significativos que se busca con esta investigación y que si bién no hace referencia directa al adobe, si nos muestra las razones de fondo que llevan a las autoridades políticas y/o religiosas de la época a resolver una situación que amerita un análisis profundo para tomar una decisión adecuada pero que si embargo fueron otros los factores que primaron, en este caso intereses económicos. Barros Arana (2000, p. 319) cita textual la carta del oidor de la Real Audiencia don Nicolás Polanco de Santillana al Rey en junio de 1647 y comenta,

\begin{abstract}
"Quiso la ciudad en cabildo abierto, movidos del horror de ver que sus mismas casas habían conspirado contra la vida de sus dueños, y eran ya sepulcros de ellos, y desmayada de poder remover tanto desmonte como ocupaban los sitios que fueron antes edificios de su vivienda, mudarse y salir como huyendo de su propia hacienda a buscar otro lugar donde poblarse, en que comenzaron a discurrir utilidades para su mudanza. Concurrimos (los oidores) en la plaza con el Obispo, todos los ministros reales, prelados de religiones, cabildo eclesiástico y secular, donde se confirió largamente el sí y el no, y se resolvió no convenir por entonces sino repararse contra el viento cada uno como mejor pudiese, y cuidar de reservar del hurto las alhajas, vestidos y los materiales desunidos, y buscar alivios de conservarse y no perderse, y amparar las monjas, las religiones, los pobres, los huérfanos, los desvalidos, y componer la república de modo que no se acabase totalmente". Esta resolución que se creería inspirada por el apego de los pobladores al suelo en que habían nacido y vivido, obedecía, sin embargo, a sentimientos de otro orden. Casi todos los solares de la ciudad estaban gravados con fuertes censos a favor de los conventos y de otras instituciones religiosas que procuraban a éstos una renta considerable. La traslación de la ciudad, dejando sin valor alguno esos solares, habría producido su abandono definitivo y privado a los conventos de una buena parte de sus entradas. La Audiencia, obedeciendo a las ideas religiosas de la época, apoyó decididamente al Obispo y a los frailes en sus gestiones; y quedó resuelto que la ciudad se reconstruiría en el mismo sitio."

[...] "Efectivamente con el terremoto de 1647 la ciudad quedó arruinada por completo y se inició una nueva etapa constructiva. Se eligieron con mayor cuidado los materiales y se emplearon nuevos elementos destinados a asegurar la estabilidad, como por ejemplo pilares y cimientos enormes que influyeron en el aspecto exterior.

Por esta misma época, La Compañía de Jesús impulsó un auge en la construcción, formando a albañiles, carpinteros, ebanistas, mecánicos, entre otros oficios, que elevaron la calidad de los sistemas constructivos y decorativos aplicados no sólo a edificios religiosos sino a todo el conjunto arquitectónico."
\end{abstract}

- 13 de febrero de 1812. Se crea y publica el primer periódico nacional "La Aurora de Chile”. Este hecho, desde el punto de vista de la investigación, constituye un hito histórico importante por cuanto es el primer medio de comunicación nacional. 
Figura 6. Aurora de Chile

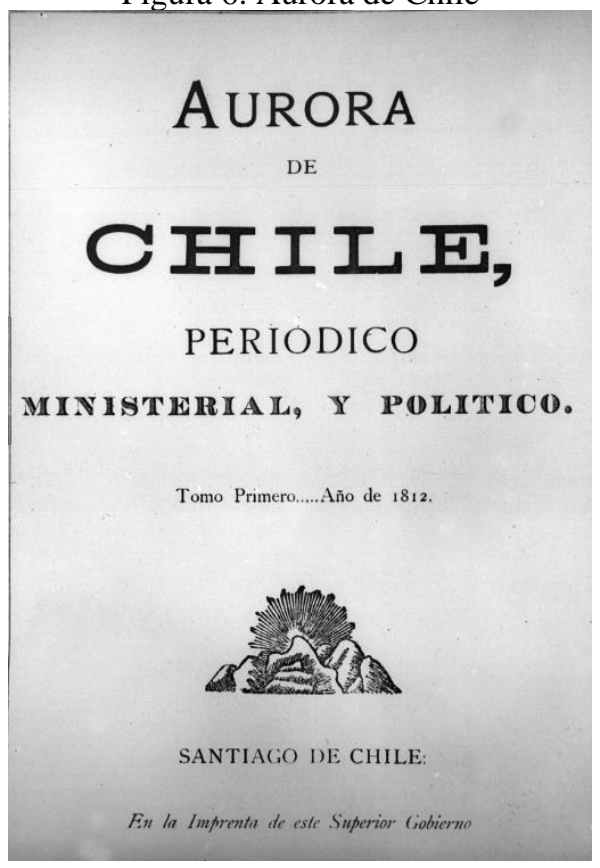

Biblioteca Nacional

- 16 de agosto de 1906. A raiz de este evento telúrico, se crea en Chile el Servicio Sismológico de Chile, como da cuenta el Boletín del mismo servicio, (1909,p. 1)

\begin{abstract}
"El terremoto de Valparaíso del 16 de Agosto de 1906 sacudió a la opinión pública de Chile i demostró a todos, tanto en la nación como en las esferas gubernativas, la necesidad absoluta de desarrollar en este país el estudio de los fenómenos sísmicos, tal vez su rasgo natural más característico. Se entendió también i vivamente la importancia de la, vulgarización de los métodos para construir asísmicamente, una parte de la sismólojía hasta la fecha completamente ignorada en Chile, lo que orijinó en gran parte los daños causados por el terrible suceso."
\end{abstract}

Además, al referirse a la "vulgarización de los métodos para construir asísmicamente" se refiere a promover la difusión y enseñanza del arte de construir en los paises sísmicos. Por esta razón, el entonces Rector de la Universidad, Sr. Valentin Letelier, como jefe de la enseñanza superior, propuso al Consejo de Instrucción Pública la creación de este Servicio y la contratación de un sismólogo europeo que, además de su trabajo científico debía encargarse de impartir clases al respecto a los estudiantes de arquitectura e ingeniería.

- 10 de noviembre de 1922. Terremoto en la zona norte de Chile, La Serena, Coquimbo. El presidente de la época, Sr. Arturo Alessandri, visita la zona afectada, sus impresiones con respecto a este viaje quedaron plasmadas en el Album Gráfico del Terremoto del Norte (Ortíz, 1923. p.4) en donde expresa que,

"Es obvia la conveniencia de utilizar esta triste oportunidad como la única ocasión que se presenta para procurar que la reconstrucción, se realice sobre las bases modernas y con materiales adecuados, dentro del propósito que las futuras habitaciones ofrezcan la posible resistencia en 
circunstancias análogas a las de la reciente conmoción. Los técnicos que me han acompañado durante la visita al norte estiman relativamente fácil la solución para el porvenir.”

Claramente existe una intención de renovación y modernización de parte de la autoridad máxima y sus técnicos pero también es evidente que los asesores técnicos en ningún momento pretendieron hacer una investigación con el objeto de rescatar lo existente y determinar por que colapsaron las edificaciones. Fue más fácil, como lo manifiesta el mismo presidente Alessandri, sentar esperanzas en nuevos materiales y en "la posible resistencia" de éstos, osea, tampoco parecía seguro de que así fuese.

En el mismo documento se señala que parte de la comitiva de carácter técnico estaba integrada por el Director General de Obras Públicas, Ingeniero Guillermo Illanes y el Ministro de Industria y Obras Públicas, Ingeniero Miguel Letelier.

Un documento bastante interesante con respecto a las construcciones dañadas por este sismo, es el Boletín del Servicio Sismológico de Chile del año 1992, de cual se desprenden observaciones muy interesantes que dan cuenta de que parte del problema son también los suelos y la poca conservación o mantención de las edificaciones.

- 1 de diciembre de 1928. Un fuerte sismo produce la destrucción casi completa de la ciudad de Talca. En un texto de un periódico local se lee, ${ }^{1}$

\begin{abstract}
"Este terremoto repite la lección que desde hace siglos nos viene dando la tierra en sus convulsiones: que debemos construir las casas teniéndolas en cuenta.

Antes parecía más difícil, pero no imposible: el adobe se llevó la preferencia para casas bajas; y aun se construyeron algunas de dos pisos de muros gruesos de tierra que han resistido todos los terremotos.

Mas ahora, con el cemento armado, la estructura de fierro y otros medios, hay facilidad para construir en forma asísmica.

Se necesita, no obstante, una ley que establezca las características de las construcciones en relación con su precio y altura. Desde luego, una ordenanza sobre escalas, en vista de las desgracias por incendios, no ha sido obedecida. Se requiere, pues, una ley que obligue a todos a construir contra terremotos."
\end{abstract}

Declara abiertamente la falta de toma de conciencia del tema sísmico en la construcción de la época y destaca la calidad resistente del adobe aunque por otra parte promueve la construcción con concreto armado y finalmente plantea la necesidad de regulaciones normativas.

- 25 de noviembre de 1935. Luego de una prórroga de un año, por Decreto 4882 de fecha 20 de noviembre de 1935, el Presidente Alessandri decreta la entrada en vigencia de la

\footnotetext{
${ }^{1}$ Tomado de un fragmento de un periódico local sin identificar, se ubica en http://www.memoriachilena.cl/602/w3-article71745.html
} 
“Ley y Ordenanza General sobre Construcciones y Urbanización” (LGCU y OGCU) aprobada por el DFL-345 del 20 de mayo de 1931. (Biblioteca del Congreso Nacional de Chile)

Esta primera OGCU, en el Capítulo I, Clasificación de los edificios, en su Art. 3.0 define nueve clases desde la A a la I atendiendo a su sistema constructivo. La primera mención del adobe la hace en la penúltima, en la "Clase H.- Edificios de madera" y el adobe sólo figura como una alternativa de material de relleno, lo que se ha llegado a denominar en Chile como Adobe en pandereta. Finalmente, la segunda mención la hace en la última, la "Clase I.- Edificios de Adobe" señalando las siguientes exigencias:

\footnotetext{
"Estos no podrán tener más de un piso, ni mayor altura de 4 metros, elemento alguno que sobresalga de la techumbre fuera de cañerías de ventilación u otros elementos análogos, ni soportar altos de ninguna especie. En casos especiales y siempre que, se consulten espesores mayores de $0.60 \mathrm{~m}$. la autoridad podrá autorizar muros de altura superior a $4 \mathrm{~m}$.

Los muros exteriores serán por lo menos de $0.60 \mathrm{~m}$. de espesor y los muros interiores o divisorios de $0.30 \mathrm{~m}$; si su longitud no fuere mayor de $5 \mathrm{~m}$.

Cuando la extensión del edificio exceda en algún sentido de $12 \mathrm{~m}$. deberán consultarse muros interiores de $0.60 \mathrm{~m}$. de espesor a distancias no mayores de $12 \mathrm{~m}$.

Cuando la longitud de los muros de $0.30 \mathrm{~m}$. de espesor exceda de $5 \mathrm{~m}$. éstos deberán reforzarse con pilares de adobe de $0.60 \mathrm{~m}$. x $0.60 \mathrm{~m}$., colocados a distancias no mayores de $4 \mathrm{~m}$. entre sí.

Los dinteles se construirán con vigas de madera que abarquen todo el espesor del muro; estas vigas tendrán $0.15 \mathrm{~m}$. de altura mínima y deberán penetrar de $0.40 \mathrm{~m}$. en cada jamba.

Los paños de paredes entre los vanos deben tener a lo menos $1.20 \mathrm{~m}$. de longitud y ningún vano se establecerá a menos de un metro del cruce de muros.

La techumbre debe apoyarse adecuadamente en los muros por medio de llaves.

No es permitida la construcción de muros de adobones en ninguna parte de un edificio. En las casas de adobes no es permitido el empleo de tabiques divisorios de esqueleto de madera con relleno de adobes colocados de canto, se prohíbe igualmente el empleo de antetecho."
}

En este mismo cuerpo legal, además de mencionar el adobe en otros artículos sobre detalles no menos interesantes, vale la pena dar una mirada al Art. 172 donde señala que "en los edificios de muros de adobe, sólo se permitirá el empleo, de techumbres livianas, cuyas acciones de apoyo sean bien centradas y verticales." Esta obligación constituye un grave error puesto que ya es sabido y ha sido materia de varias invetigaciones que uno de los factores importantes en el sistema constructivo con adobes es el peso de la techumbre en donde la teja de arcilla aporta una buena parte de éste. Pero si un cuerpo legal como lo es la OGCU exige o condiciona su autorización al uso de materiales livianos es lógico pensar que las personas usarán cubiertas más livianas y que para estos años las alternativas eran al parecer, pocas, más que nada planchas metálicas.

- 1939. En este año se produce otro hito importante a nivel nacional, tras varios años de la Gran Depresión (1929-1932) en donde Chile fue la nación más devastada según un informe de la liga de las Naciones (World Economic Survey) y sumado a una crisis 
político - económica de varios años, finalmente, en el gobierno de Pedro Aguirre Cerda y en gran medida impulsado por el terremoto de Chillán de este mismo año, se creó la Corporación de Fomento de la Producción (CORFO) con el fin de impulsar la industrialización nacional, producto de esto se crea entre otras industrias la Compañía de Acero del Pacífico (CAP).

Es inegable que la situación económica por la que atravesaba Chile en esos años requería talvez de medidas como las que se tomaron desde el punto de vista productivo, pero asiste la duda si las exigencias del Art. 172 de la OGCU mencionado anteriormente, fue también producto de una decisión técnica o netamente económica como una manera de fomentar la producción.

\section{CONSIDERACIONES FINALES}

Es importante reiterar que esta investigación está en una primera etapa de análisis histórico que recién comienza y que queda mucho material por revisar, por lo tanto los resultados presentados son sólo las primeras aproximaciones parciales que si bien no son totalmente concluyentes pero si dan indicios de respuestas para una mejor comprensión del problema.

Desde los inicios pre hispánicos ya se vislumbran intercambios culturales entre los nativos sudamericanos, especificamente en Chile por la expansión del Imperio Inca y que han influido en las características constructivas y de materialidad de sus edificaciones, sobre todo en la zona norte. Luego con la llegada de los españoles, se ve como se produce un mestizaje entre una cultura local sísmica con otra foranea que no está acostumbrada a los movimientos telúricos, al menos en la magnitud que se dan en Chile. Se crean los primeros asentamientos urbanos que en sus inicios tienen que lidiar con dos grandes fuerzas; la resistencia indígena y los constantes terremotos. Esta situación de inestabilidad genera que las primeras construcciones tengan un carácter más efimero y provisorio. De acuerdo a los antecedentes estudiados, se ve que el adobe en épocas pre hispánicas practicamente no se utilizaba en la zona centro sur del pais y que su uso comienza cuando toman formas las ciudades y se expande hacia la zona sur.

En estas ciudades y producto de su natural desarrollo, en su inicios acogen al sistema constructivo con adobes pero al pasar de los años, condicionados por los permanentes desastres naturales, las autoridades encuentran en estos sucesos la oportunidad de producir cambios basados principalmente en intereses económicos que van menospreciando y abandonando al adobe. La más clara expresión de lo anterior hasta el momento se puede ver en la promulgación de la Ley General de Construciones y Urbanismo (LGCU) y la Ordenanza General de Construcciones y Urbanismo (OGCU). 


\section{AGRADECIMIENTOS}

El autor agradece el permanente e incondicional apoyo de la Facultad de Arquitectura, Urbanismo y Geografía de la Universidad de Concepción, Chile. 


\section{REFERENCIAS BIBLIOGRAFICAS}

Amunátegui, M. (1882). El Terremoto del 13 de Mayo de 1647. Santiago, Chile. R. Jover Editor. p. 526 y 527

Barros Arana, D.(2000,). Historia General de Chile, (Edición original 1884), Santiago, Chile, tomo I, p. 282. Ed. Universitaria

Barros Arana, D.(2000,). Historia General de Chile, (Edición original 1884), Santiago, Chile, tomo IV, p. 319. Ed. Universitaria

Conde de Montessus de Ballore. (1909) Boletin del Servicio Sismilójico de Chile I. Santiago. Chile. p.1. Imprenta Cervantes.

Del Busto, J. A. (1981). Perú Incaico, tercera edición, Lima, Perú. p. 274 y 276.

Jorquera, N. (2014). Culturas Sísmicas: Estrategias vernaculares de sismorresistencia del patrimonio arquitectónico chileno.

Ortíz, M. J. (1923). Album Gráfico del Terremoto del Norte. Santiago. Chile. p. 4. Editorial Universitaria.

Vergara, T. (2000). Tahuantinsuyo: El mundo de los Incas. En Teodoro Hampe Martínez. Historia del Perú. Incanato y conquista. Barcelona: Lexus. ISBN 9972-625-35-4. 